Gabriela Ręka, Emilia Nowak, Dominika Psiuk, Agata Rocka, Halina Piecewicz-Szczęsna. The use of fecal microbiota transplantation in the treatment of intestinal diseases. Journal of Education, Health and Sport. 2020;10(9):676-689. eISSN 23918306. DOI http://dx.doi.org/10.12775/JEHS.2020.10.09.082

https://apcz.umk.pl/czasopisma/index.php/JEHS/article/view/JEHS.2020.10.09.082 https://zenodo.org/record/4047945

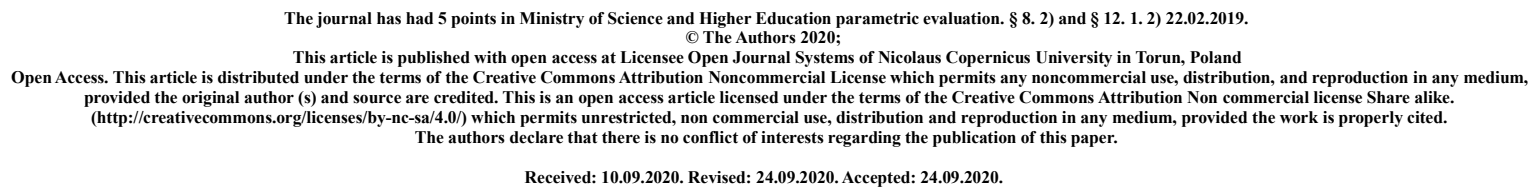

\title{
The use of fecal microbiota transplantation in the treatment of intestinal diseases
}

\author{
Gabriela Ręka ${ }^{1}$, Emilia Nowak ${ }^{1}$, Dominika Psiuk ${ }^{1}$, Agata Rocka ${ }^{1}$,
} Halina Piecewicz-Szczęsna²

\author{
${ }^{1}$ Gabriela Ręka \\ https://orcid.org/0000-0001-9728-5281 \\ gabrysia.reka@gmail.com \\ ${ }^{1}$ Emilia Nowak \\ https://orcid.org/0000-0003-4012-2419 \\ emilia.m.nowak@wp.pl \\ ${ }^{1}$ Dominika Psiuk \\ https://orcid.org/0000-0003-3319-3489 \\ dominika.psiuk@gmail.com \\ ${ }^{1}$ Agata Rocka \\ https://orcid.org/0000-0003-4738-3160 \\ agatarocka2@gmail.com \\ ${ }^{2}$ Halina Piecewicz-Szczęsna \\ https://orcid.org/0000-0002-0573-7226 \\ halpiec@gmail.com
}

\footnotetext{
${ }^{1}$ Students' Scientific Association of Chair and Department of Epidemiology and Clinical Research Methodology, Medical University of Lublin, Radziwiłłowska 11, 20-080 Lublin, Poland
} 
${ }^{2}$ Chair and Department of Epidemiology and Clinical Research Methodology, Medical University of Lublin, Radziwiłłowska 11, 20-080 Lublin, Poland

\section{Introduction and purpose}

Fecal microbiota transplantation (FMT) is a process of transferring gut microflora from a healthy donor to a recipient. It is indicated that the human distal gastrointestinal tract consists of more than 1000 species of bacteria, which have an impact on local and systemic processes related to immunity, nutrition and the gut-brain axis. The growth of new bacterial species, reduction of microbial variety or big changes in the ratio between them can lead to a dysbiosis. The relationship between intestinal microbiota and some systemic diseases is seen. The study aimed to present the state of knowledge on FMT in the treatment of intestinal diseases. Publications from the last 5 years from the Pubmed database were included.

\section{A brief description of the state of knowledge}

Intestinal diseases constitute the main group of indications for FMT. FMT can be delivered via upper or lower gastrointestinal routes. The review presents different studies concerning the usage of FMT. Among them, recurrent Clostridium difficile infection, colitis ulcerosa, Crohn's disease and irritable bowel syndrome are mainly indicated in the literature. FMT might find its application also in a treatment of multidrug-resistant organisms colonization, antibiotic-associated diarrhea, gut acute graft-versus-host disease and radiation enteritis. There is a possibility of the occurrence of adverse events from the digestive tract after FMT. However, the studies evaluate them as mostly mild and transient. In some cases it was necessary to repeat FMT to achieve a satisfactory final result.

\section{Conclusions}

Fecal microbiota transplantation gives hope for a safe and effective method of treatment of certain intestinal diseases. There is a need for conducting further analysis of the method.

Key words: fecal microbiota transplantation; gastrointestinal microbiome; intestinal diseases

\section{Introduction and purpose}

Fecal microbiota transplantation (FMT) is a process of transferring microflora from the gut of a healthy donor into the digestive tract of a recipient [1]. It is commonly called a stool transplantation and nowadays a lot of research on this topic is coming up. Fecal transplantation has been known since the 4th century when human fecal suspension by mouth was used to treat serious diarrhea and food poisoning in China. There are some undocumented reports of the use of parental feces to treat antibiotic-associated diarrhea in their children. However, the first confirmed case of Clostridium difficile infection treated with FMT was reported in 1983 by Schwan et al. [2]. Fecal material can be delivered via upper or lower gastrointestinal routes. It is indicated that the human distal gastrointestinal tract consists of more than $10^{14}$ bacteria of about 1000 species [1]. Intestinal microbiome mainly contains species from the phylum Bacteroidetes, Firmicutes, Actinobacteria, and less Proteobacteria. These microorganisms have an impact on local and systemic processes, like maturation of mucosal immunity, vitamin delivery, nutrient transformation, communication between gut and brain, and progression of the tumor [3]. The appropriate function of the 
intestine microbiota relies on a stable cellular composition. The expansion of new bacterial species like Proteobacteria, reduction of microbial variety or big changes in the ratio between normal phyla might lead to an imbalance called dysbiosis [3]. Over time, scientists get a better understanding of the relationship between systemic diseases and intestinal microbiota. Despite reports on the possibility of treating diseases of many organs using the transplantation of fecal microbiota, intestinal diseases, especially recurrent Clostridium difficile infection and inflammatory bowel disease, still constitute the main group of indications.

The objective of the study is to present the current state of knowledge on fecal microbiota transplantation in the treatment of intestinal diseases. The newest publications from the last 5 years available on the Pubmed database were taken into account.

\section{Description of the state of knowledge}

\section{Description of the process of microbiota transplantation}

Because of a risk of transmission of infectious pathogens via FMT, candidate donors must be carefully tested. Potential donors should be questioned in detail in a medical interview. The donor should be tested serologically towards Amoeba, Treponema pallidum and viruses (HAV, HBV, HCV, HIV, CMV, EBV) and undergo stool screening towards rotavirus, enterovirus, norovirus, adenovirus, astrovirus, Clostridium difficile, Shigella, Salmonella, Campylobacter, Yersinia, Helicobacter pylori, enteropathogenic Escherichia coli, Giardia, Cryptosporidium and others [1,2,4]. Fecal microbiota may be sourced from relatives of candidate donors or from universal donors through stool banks [2,4]. It depends on the indication, for example donor should be unrelated when FMT is being used to treat diseases where genetics play a contributing role, such as inflammatory bowel disease [2]. Fecal material must be diluted and homogenized before its administration [2]. Preparation in the laboratory is crucial for an effective fecal microbiota transplantation and the process can be divided into "rough filtration", "filtration plus centrifugation", and "microfiltration plus centrifugation" [5]. It is reported that some preparation methods can damage the content of living fecal microbes, that is why shortening the time from defecation to infusion or from defecation to freezing to one hour highly improves the clinical response and costeffectiveness of FMT, particularly for patients with inflammatory bowel disease [5]. Before transplantation, a bowel lavage with a colon preparation agent should be applied. The possibilities of delivering fecal material include swallowing oral capsule, administering the material via nasogastric tube, nasoduodenal tube, esophagogastroduodenoscopy, colonoscopy or retention enema [1,4]. Despite the fact that an upper gastrointestinal route can be simply performed, some problems, like uncomfortable feeling, less volume of infusion, and risk of vomiting and aspiration, can occur. Other possible side effects include abdominal tenderness, diarrhea, constipation, flatulence, fever, peritonitis, pneumonia, flare up of inflammatory bowel disease, or endoscopic complications such as perforation and bleeding [1].

\section{The use of FMT in recurrent Clostridium difficile infection}

Clostridium difficile infection (CDI) is a major source of morbidity and mortality for hospitalized patients. Although most patients have a clinical response to existing 
antimicrobial therapies, recurrent infection develops in up to $30 \%$ of patients. FMT is a novel approach to this complex problem [6].

Hagel et al. carried out a long-term retrospective multicenter observational trial to investigate the clinical effectiveness of FMT for the treatment of recurrent CDI. The study involved 133 patients and the FMT was administered via duodenal $(\mathrm{n}=59)$, colonic $(\mathrm{n}=55)$, gastric $(n=4)$ route or by oral capsules $(n=13)$. Primary cure on 30 day 30 was achieved in $84.2 \%(n=101 / 120)$ and on 90 day in $78.3 \%(n=72 / 92)$ of patients. Eighteen patients needed re-treatment and one patient received 4 FMT procedures. A total of $12 \%$ of patients experienced adverse events, which might be related with the procedure, including: nausea, fever, abdominal pain, belching, emesis, throat/retrosternal discomfort, aspiration pneumonia (acquired during endoscopy), loss of a tooth (during endoscopy), irritable bowel syndrome, and hemorrhage (during endoscopy), but none of these patients died within the following 30 days. This study proved the safety and effectiveness of FMT in CDI treatment [7].

The aim of Kelly et al. clinical trial was to determine the efficacy and safety of FMT for treatment of recurrent CDI. This study involved 46 patients, who had 3 or more recurrences of CDI and received a full course of vancomycin for their most recent acute episode. Patients were randomly assigned to the group that received FMT with donor stool (heterologous) $(n=22)$ or group with patient's own stool (autologous) $(n=24)$ administered by colonoscopy. The study showed that $90.9 \%$ in the donor FMT group achieved clinical cure compared with $62.5 \%$ in the autologous FMT group. Moreover, all 9 patients who had recurrence after autologous FMT subsequently crossed over to treatment with donor FMT and were free of further CDI. No FMT-related serious adverse events were observed and all FMT donors restored gut bacterial community diversity and composition. This prospective, double-blind, randomized, controlled trial proved safety and effectiveness of heterologous FMT in preventing CDI episodes [8].

Hvas et al. compared the effectiveness of FMT with the effects of fidaxomicin and vancomycin treatment. This study involved 64 patients. Patients were randomly assigned to three groups: a group that received FMT, applied by colonoscopy or nasojejunal tube, after 410 days of vancomycin (125 mg 4 times daily; $\mathrm{n}=24$ ), a group that received fidaxomicin for 10 days (200 mg twice daily; $\mathrm{n}=24)$, or a group that received vancomycin for 10 days (125 mg 4 times daily; $n=16$ ). The primary outcome, which included clinical resolution and a negative result from a polymerase chain reaction test for Clostridium difficile toxin 8 weeks after the treatment, was observed in $71 \%$ of patients in FMT group, 33\% of patients in fidaxomicin group and $19 \%$ of patients in vancomycin group. Overall, clinical resolution occurred respectively in $92 \%, 42 \%$ and $19 \%$ of patients. Patients who had recurrent CDI after this course of treatment were offered rescue FMT and there was no significant difference between patients who received FMT as their initial therapy and patients who received rescue FMT. There was 1 serious adverse event in the form of a sepsis-like clinical picture with pyrexia, convulsions, vomiting, and diarrhea that might have been related to FMT, but the patient had complete recovery within 24 hours without further treatment. The study showed that the FMT is more effective than fidaxomicin or vancomycin in recurrent CDI treatment [9].

Hota et al. compared 14 days of oral vancomycin followed by a single FMT by enema $(n=16)$ with 6 weeks of oral vancomycin $(n=12)$ in patients with acute recurrence of CDI. 
Remission was observed in $43.8 \%$ of patients in FMT by enema group and in $58.3 \%$ of patients in oral vancomycin group. There was no significant difference in reducing recurrent CDI between the groups. Although, fecal microbiota analysis of 3 successful FMT patients demonstrated increased diversity [10].

Ianiro et al. researched the efficacy of FMT in patients with severe CDI refractory to antibiotics. Fifty-six patients were randomly assigned to one of the two groups: receiving a single fecal infusion via colonoscopy followed by a 14-day vancomycin course $(n=28)$ or multiple (at least two) fecal infusions with a 14-day vancomycin course $(n=28)$. Remission was observed respectively in $75 \%$ and $100 \%$ of patients. This trial improved that multiple fecal infusions with vancomycin was significantly more effective than a single fecal transplant followed by vancomycin in curing severe Clostridium difficile infection refractory to antibiotics [11].

Lee et al. conducted a randomized, double-blind, noninferiority trial to determine whether frozen-and-thawed FMT is noninferior to fresh FMT in terms of clinical efficacy among patients with recurrent or refractory CDI. Patients were randomly allocated to receive frozen $(\mathrm{n}=114)$ or fresh $(\mathrm{n}=118)$ FMT via enema. The primary outcome was clinical resolution of diarrhea without relapse at 13 weeks. This trial showed that the use of frozen compared with fresh FMT did not result in lower efficiency in suspending diarrhea in patients with recurrent or refractory CDI. There were also no differences in the proportion of adverse or serious adverse events between the treatment groups [12].

On the other hand, Kao et al. investigated whether the clinical effectiveness differs depending on the way of FMT administration. This noninferiority, unblinded, randomized trial involved 116 patients with recurrent CDI, who were randomly assigned to FMT by capsule $(n=57)$ or by colonoscopy $(n=59)$. Prevention of recurrent CDI up to 12 weeks after FMT was achieved in $96.2 \%$ in both groups. Rates of minor adverse events were $5.4 \%$ for the capsule group and $12.5 \%$ for the colonoscopy group. Between groups there was no significant difference in improvement in quality of life measured by the 36-Item Short Form Survey, but in group receiving FMT by capsules compared with FMT by colonoscopy, a significant more patients rated their experience, on scale of 1 (not at all unpleasant) to 10 (extremely unpleasant), as "not at all unpleasant" (respectively 66\% vs. 44\%). This study proved that FMT via oral capsules is not inferior to delivery by colonoscopy in recurrent CDI treatment [13].

Jiang et al. also investigated the safety and preliminary efficacy of orally administered lyophilized microbiota products compared with frozen products by enema. Sixty-five patients, who had 3 or more recurrences of CDI, were randomized to receive encapsulated lyophilized fecal microbiota $(n=31)$ or frozen FMT by enema $(n=34)$. CDI recurrence was prevented in $84 \%$ of patients in the group of oral capsules and in $88 \%$ patients receiving FMT by enema ( $p$ $=0.76$ ). Both products normalized fecal microbiota diversity, but the lyophilized orally administered product was less effective in repleting Bacteroidia and Verrucomicrobia classes compared to frozen product via enema. This study showed that any of the ways of delivery, oral or rectal, did not influence adverse events in FMT and both are comparably efficient in recurrent CDI [14].

Kim et al. carried out a retrospective study to compare cost and scheduling efficiency of FMT by universal donors to patient-directed donors. A total of 111 fecal microbiota 
transplantations were performed on 105 patients, including 56 FMT from patient-directed donors and 55 from universal donors. This analysis showed that total consultation fees and costs for donor screening and stool preparation were significantly lower in the universal donor group. Also time from consultations to infusion was shorter in the universal donor cohort, while recurrences within 8 weeks after FMT and frequency of adverse events were equivalent. The use of universal donors resulted in significant cost savings and scheduling efficiency, whereby the effectiveness of CDI treatment was comparable to patient-directed donors [15].

\section{The use of FMT in ulcerative colitis}

Ulcerative colitis (UC) is a chronic inflammatory disease of mucous membrane and submucosa with yet unknown etiology. Development of disease is influenced by both genetic and environmental factors. UC is unpredictable and demonstrates periods of exacerbation and remission [16]. Incidence of disease is the highest among people in the third and fourth decade of life. In Europe UC is affecting about 505 out of 100000 people, in the USA 214 out of 100000 and incidences in Australia can be rated as 17.4 out of 100000 . Unfortunately, the incidence of the disease is constantly increasing globally. The most common symptoms are blood in the stool and diarrhea, urgency, incontinence, fatigue, increased frequency of defecation and abdominal discomfort. In late stage weight loss and high fever could occur [17].

The factors that contribute to ulcerative colitis, disrupt the microbiological ecosystem of the colon by breaking the epithelial barrier. This allows the intestinal microorganisms to interact with the immune cells. It causes an inflammatory reaction, which is no longer controlled. Over time, extensive damage to the mucous membrane occurs, which spreads to other parts of the colon. So far, there is no fully effective treatment for ulcerative colitis. Anti-inflammatory drugs are mainly used to relieve the mild symptoms. In advanced forms, the therapy is based on steroid or immunomodulating drugs. However, many patients do not receive a permanent remission. In approximately $15 \%$ of patients with ulcerative colitis, within 20 years after diagnosis, it is necessary to surgically remove a large section of the colon, or even all of it [18]. That is why it is so important to search for new effective treatment methods.

Recently FMT was introduced in UC treatment. Clinical trials carried out so far demonstrated that using FMT is a promising therapeutic method as it induces disease remission and is safe to use [19]. There was a meta-analysis in which 277 patients from 4 trials were involved. The results showed disease remission in $42.1 \%$ patients who received FMT and $22.6 \%$ in the control group. The frequency of adverse events was comparable in both FMT and control groups (7.1\% and 5.1\% respectively) [20]. Other clinical trials were carried out and demonstrated FMT efficacy as well. Paramsothy et al enrolled 81 patients with UC and performed FTM in 41 patients, the rest of participants were placebo-controlled group. In the FMT group clinical remission and improvement in colonoscopy were observed in 11 of 41 patients and in 3 of 40 patients from the placebo group. Many adverse effects were observed in both groups (78\% and $83 \%$ respectively), serious adverse events occurred in two FMT patients and one placebo patient. Moreover, fecal samples were collected before and after performing FMT and analyzed. Microbial diversity was greater in samples from 
patients who achieved remission compared with patients who did not and consisted of two new species - Eubacterium hallii and Roseburia inulivorans [21,22]. Mahajan et al. described FMT efficacy in reducing adverse effects of corticosteroid therapy, for example weight loss (from BMI $28.0 \mathrm{~kg} / \mathrm{m}^{2}$ to $23.5 \mathrm{~kg} / \mathrm{m}^{2}$ without diet modifications), reducing hair loss, backache, morning stiffness and allergic rhinitis after 22 weeks of 7 FMT sessions [23]. Other studies demonstrated that FMT could reduce disease symptoms, for example diarrhea, stomach ache and blood in the stool, however decrease of C-reactive protein level was not observed [24]. The FMT method showed efficacy in treatment-resistant UC as well and clinical, endoscopic and laboratory remission was observed in $43.3 \%$ patients [25].

Promising results in described above studies show that performing FMT could be efficient for patients with UC. However, more detailed research and long-term analyses are required to finally confirm its effectiveness.

\section{The use of FMT in Crohn's disease}

Crohn disease is another chronic inflammatory bowel disease with unknown pathogenesis. It affects the entire gastrointestinal tract, in which patchy, transmural lesions are spread. The most common symptoms are abdominal pain, diarrhoea, rectal bleeding, weight loss and fatigue and appear in periods of activity and remission [26,27]. Pharmacotherapy is based on immunomodulatory and biological agents, such as azathioprine, 6-mercaptopurine, methotrexate and infliximab, adalimumab, certolizumab. However, they demonstrate many side effects and often lack of efficacy $[28,29]$.

There was a study which aimed to evaluate the frequency of adverse effects and efficacy of fecal microbiota transplantation (FTM) in 139 patients with Crohn's disease (CD). During 1 month after FMT perfomation $13.6 \%$ of mild adverse effects were observed, including increased frequency of defecation, fever, abdominal pain, flatulence, hematochezia, vomiturior, bloating and herpes zoster. However, after the first month no adverse effects occurred. The frequency of FMT was higher in patients who underwent manual method than automatic method $(21.7 \%$ and $8.7 \%$ respectively). In this study, no clinical efficacy of FMT in patients with CD was observed [30]. There were two open-label studies involving 19 and 10 patients with CD and treated with FMT. In these studies 11 out of 19 patients and 3 out of 10 patients demonstrated a clinical response, in both measured with Harvey-Bradshaw Index. First study showed that FMT was safe and no serious adverse events were observed. However, in the second study 2 out of 10 patients they occured in significant level [31,32]

Sixty-nine patients with CD who underwent and responded to FMT in the past were enrolled in Pan Li et al. study. This study aimed to evaluate the optimal timing for performing FMT second time to maintain the long-term clinical effects in patients with CD. The median time of maintaining clinical response to the first FMT in total 69 patients was 125 days and to the second FMT - 176.5 days. The results showed that patients with CD could be administered the second course of FMT less than 4 months after the first FMT for maintaining the clinical benefits from the first FMT [33].

In a pilot randomized controlled study by Sokol et al. 17 patients with exacerbation of the $\mathrm{CD}$ received oral corticosteroid and then, during the clinical remission, they were randomized to receive either FMT $(n=8)$ or sham transplantation $(n=9)$ via colonoscopy. None of the patients reached the primary endpoint, which was the implantation of the donor 
microbiota at week 6 in Sorensen index $>0.6$. The clinical remission rate at 10 and 24 weeks was $44.4 \%$ and $33.3 \%$ in the sham transplantation group and $87.5 \%$ and $50.0 \%$ in the FMT group. Moreover, 6 weeks after the procedure, in the FMT group compared to the sham transplantation, the Crohn's Disease Endoscopic Index of Severity decreased and the CRP level increased. This study proved that the maintenance of CD remission is associated with higher colonization by donor microbiota [34].

\section{The use of FMT in irritable bowel syndrome}

Irritable bowel syndrome (IBS) is a common chronic gastrointestinal disease affecting an estimated $11 \%$ of the adult population [35]. IBS is associated with disorders of intestinal function, with no structural or biochemical disruptions, so the disease can not be detected by using routine diagnostic tests. Disease symptoms are abdominal pain and discomfort, abnormalities in stool and flatulences [36]. Etiology of IBS is yet unknown; however, it is considered that it results from many factors. Current therapy is complex and includes lifestyle and diet modifications, using probiotics and pharmacotherapy. This multi-component approach has made great progress in IBS treatment; however, due to various adverse effects it is needed to develop new methods [37]. It is thought that FMT can positively influence patients with IBS.

The study conducted by Mazzawi et al. showed improvement in quality of life and reduction of IBS symptoms for 28 weeks after performing FMT. Thirteen patients and thirteen healthy people were involved and fecal samples were collected from both groups before and 1, 3, 12 and 20-28 weeks after FMT. Before FMT there were significant differences in the profile of bacterias Ruminococcus gnavus, Actinobacteria i Bifidobacteria in the stool between two groups. After 3 weeks after FMT the differences were unnoticeable. Moreover, in 12 and 20-28 week after FMT in patients' samples new strains of bacteria were noticed - Bacteroides / Prevotella, Alistipes, Actinobacteria i Bifidobacteria [38,39].

No satisfactory results in IBS treatment were reported in a randomized double-blind study by Halkjær et al. with 52 patients of various stages of the IBS (moderate to severe). The results showed that the use of FMT changes the intestinal microflora in IBS patients. However, 3 months after treatment, placebo patients reported a greater change in symptom relief compared to FMT. This study showed that the change in an intestinal microbiota was not sufficient to achieve a clinical improvement in IBS patients [40].

Another randomised double-blind study with 165 IBS patients showed a positive role of FMT. Patients were divided into three groups: placebo, a group receiving 30g FMT and $60 \mathrm{~g}$ FMT from the donor. The treatment response occurred in $23.6 \%$ of patients in the placebo group, $76.9 \%$ of patients in the $30 \mathrm{~g}$ FMT group and $89.1 \%$ in the $60 \mathrm{~g}$ FMT group. Moreover, after one month of the FMT procedure, higher concentrations of Eubacterium biforme, Lactobacillus spp. and Alistipes spp. and lower concentrations of Bacteroides spp. were observed in both groups: $30 \mathrm{~g}$ FMT and $60 \mathrm{~g}$ FMT. There was a significant clinical progression and quality of life improvement in patients receiving FMT. The majority of observed adverse events were mild, self-limited gastrointestinal symptoms (intermittent abdominal pain, diarrhea, or constipation that occurred 2 days after starting FMT therapy) [41]. 
Differences between trials' results indicate the need for further multicenter studies, with more patients, to assess the effectiveness of FMT in the treatment of IBS.

\section{The use of FMT in other intestinal disorders}

In the literature, reports about the possibility of treating other intestinal diseases with fecal microbiota transplantation can be found. Examples of other indications are presented below.

Dai et al. describes the utility of FMT for antibiotic-associated diarrhea in critically ill patients from the intensive care unit. Dysbiosis was induced by administration of antibiotics and led to diarrhea induced by $C$. difficile and other bacteria (the diagnosis could not be confirmed for all patients due to the lack of detection kit). Eighteen out of twenty severely ill patients with antibiotic-associated diarrhea who received rescue FMT were enrolled for examination. Before and during the onset of diarrhea, patients were treated with four types of antibiotics $(4.2 \pm 2.1) .44 .4 \%$ of patients recovered from abdominal symptoms without return and survived for at least 12 weeks after being discharged from the ward. $100 \%$ of abdominal pain, $86.7 \%$ of diarrhea, $69.2 \%$ of abdominal distention, and $50 \%$ of hematochezia were improved after FMT [42]. Eight deaths, which were unrelated to transplantation, occurred during this time. $38.9 \%$ of patients had transplantation-related adverse events, like increased diarrhea frequency, increased serum amylase, abdominal pain, and fever. Although FMT poses a risk of pathogen transmission from the donor, it might be a better method than probiotics for total restoration of bowel microbiome [42].

Fecal microbiota transplantation appeared in recent studies to be an alternative for decolonization of multidrug-resistant organisms [42]. Singh et al. treated 15 patients with ESBL-producing Enterobacteriaceae with FMT and proved that FMT can be an efficient treatment in patients carrying ESBL [43]. A microbial modification towards donor composition and restoration of fecal microbial variety after transplantation were shown at baseline and 4 weeks after FMT. Six out of fifteen patients were ESBL-negative after the second transplant and three out of fifteen patients at 1,2 and 4 weeks after the first transplant were negative [43].

Kakihana et al. assessed in a pilot study the safety of FMT for treating steroidresistant $(n=3)$ or steroid-dependent $(n=1)$ gut aGVHD (acute graft-versus-host disease) in stem cell transplantation [44]. 3 patients responded completely to fecal transplantation and 1 patient showed a partial response. Despite the fact that microflora infusion may be a potential risk factor for infection in severely immunosuppressed patients after stem cell transplantation, no serious adverse events attributed to FMT have been reported. Authors concluded that fecal microbiota transplantation might become an alternative to glucocorticosteroids treatment option for aGVHD by influencing the immune system and improving effects of treatment of immune-mediated enteritis such as GVHD [44].

Interestingly, there are reports on the possible treatment of radiation enteritis using FMT. It was noticed that chronic radiation enteritis is associated with dysbiosis. Ding et al. highlighted that FMT alleviated the intestinal symptoms and endoscopic mucosal harm in chronic radiation enteritis [45]. In a pilot study five female patients underwent FMT. Three patients achieved positive outcomes 8 weeks after last infusion (improvement in diarrhea, abdominal/rectal pain, rectal hemorrhage, and fecal incontinence). There were no FMT- 
related complications, like death or infections, besides one mild FMT-related transient nausea [45].

\section{Conclusions}

Fecal microbiota transplantation is a rapidly developing, promising method of restoring the gut microbiota and treatment of different intestinal diseases. Recurrent Clostridium difficile infection and inflammatory bowel disease are best known indications among intestinal diseases for FMT. The potential of FMT has not been fully understood, so there is a strong need for conducting further in-depth research concerning FMT, involving a bigger study group and particularly in less common bowel diseases.

\section{References}

1. Hsu WH, Wang JY, Kuo CH. Current applications of fecal microbiota transplantation in intestinal disorders. Kaohsiung J Med Sci. 2019;35(6):327-331. doi: 10.1002/kjm2.12069.

2. Kelly CR, Kahn S, Kashyap P, Laine L, Rubin D, Atreja A, Moore T, Wu G. Update on Fecal Microbiota Transplantation 2015: Indications, Methodologies, Mechanisms, and Outlook. Gastroenterology. 2015;149(1):223-237. doi:10.1053/j.gastro.2015.05.008.

3. GWeiss GA, Hennet T. Mechanisms and consequences of intestinal dysbiosis. Cell Mol Life Sci. 2017;74(16):2959-2977. doi: 10.1007/s00018-017-2509-X.

4. GKim KO, Gluck M. Fecal microbiota transplantation: an update on clinical practice. Clin Endosc. 2019;52(2):137-143. doi: 10.5946/ce.2019.009.

5. Zhang F, Cui B, He X, Nie Y, Wu K, Fan D, FMT-standardization Study Group. Microbiota transplantation: concept, methodology and strategy for its modernization. Protein Cell. 2018;9(5):462-473. doi: 10.1007/s13238-018-0541-8.

6. Liubakka A, Vaughn BP. Clostridium difficile infection and fecal microbiota transplant. AACN Adv Crit Care. 2016;27(3):324-337. doi: 10.4037/aacnacc2016703.

7. Hagel S, Fischer A, Ehlermann P, Frank T, Tueffers K, Sturm A, Link A, Demir M, Siebenhaar A, Storr M, Glueck T, Siegel E, Solbach P, Goeser F, Koelbel CB, Lohse A, Luebbert C, Kandzi U, Maier M, Schuerle S, Lerch MM, Tacke D, Cornely OA, Stallmach A, Vehreschild M. Fecal Microbiota Transplant in Patients With Recurrent Clostridium Difficile Infection. Dtsch Arztebl Int. 2016;113(35-36):583-9. doi: 10.3238/arztebl.2016.0583.

8. Kelly CR, Khoruts A, Staley C, Sadowsky MJ, Abd M, Alani M, Bakow B, Curran P, McKenney J, Tisch A, Reinert SE, Machan JT, Brandt LJ. Effect of Fecal Microbiota Transplantation on Recurrence in Multiply Recurrent Clostridium difficile Infection: A Randomized Trial. Ann Intern Med. 2016;165(9):609-616. doi: 10.7326/M16-0271.

9. Hvas CL, Dahl Jørgensen SM, Jørgensen SP, Storgaard M, Lemming L, Hansen MM, Erikstrup C, Dahlerup JF. Fecal Microbiota Transplantation Is Superior to Fidaxomicin for Treatment of Recurrent Clostridium difficile Infection. Gastroenterology. 2019;156(5):1324-1332.e3. doi: 10.1053/j.gastro.2018.12.019. 
10. Hota SS, Sales V, Tomlinson G, Salpeter MJ, McGeer A, Coburn B, Guttman DS, Low DE, Poutanen SM. Oral Vancomycin Followed by Fecal Transplantation Versus Tapering Oral Vancomycin Treatment for Recurrent Clostridium difficile Infection: An Open-Label, Randomized Controlled Trial. Clin Infect Dis. 2017;64(3):265-271. doi: $10.1093 / \mathrm{cid} / \mathrm{ciw} 731$.

11. Ianiro G,Masucci L, Quaranta G, Simonelli C, Lopetuso LR, Sanguinetti, Gasbarrini A, Cammarota G. Randomised clinical trial: faecal microbiota transplantation by colonoscopy plus vancomycin for the treatment of severe refractory Clostridium difficile infection-single versus multiple infusions. Aliment Pharmacol Ther. 2018;48(2):152-159. doi: 10.1111/apt.14816.

12. Lee CH,Steiner T, Petrof EO, Smieja M, Roscoe D, Nematallah A, Weese JS, Collins S, Moayyedi P, Crowther M, Ropeleski MJ, Jayaratne P, Higgins D, Li Y, Rau NV, Kim PT. Frozen vs Fresh Fecal Microbiota Transplantation and Clinical Resolution of Diarrhea in Patients With Recurrent Clostridium difficile Infection: A Randomized Clinical Trial. JAMA.2016;315(2):142-9. doi: 10.1001/jama.2015.18098.

13. Kao D, Roach B, Silva M, Beck P, Rioux K, Kaplan GG, Chang HJ5, Coward S, Goodman KJ, Xu H, Madsen K, Mason A, Wong GK, Jovel J, Patterson J, Louie T. Effect of Oral Capsule- vs Colonoscopy-Delivered Fecal Microbiota Transplantation on Recurrent Clostridium difficile Infection: A Randomized Clinical Trial. JAMA. 2017;318(20):1985-1993. doi: 10.1001/jama.2017.17077.

14. Jiang ZD, Jenq RR, Ajami NJ, Petrosino JF, Alexander AA, Ke S, Iqbal T, DuPont AW, Muldrew K, Shi Y, Peterson C, Do KA, DuPont HL. Safety and preliminary efficacy of orally administered lyophilized fecal microbiota product compared with frozen product given by enema for recurrent Clostridium difficile infection: A randomized clinical trial. PLoS One. 2018;13(11):e0205064. doi: 10.1371/journal.pone.0205064.

15. Kim KO, Schwartz MA, Lin OST, Chiorean MV, Gluck M. Reducing Cost and Complexity of Fecal Microbiota Transplantation Using Universal Donors for Recurrent Clostridium difficile Infection. Adv Ther. 2019;36(8):2052-2061. doi: 10.1007/s12325-019-00974-x.

16. Yamamoto-Furusho JK, Gutiérrez-Grobe Y, López-Gómez JG, Bosques-Padilla F, Rocha-Ramírez JL. The Mexican consensus on the diagnosis and treatment of ulcerative colitis. Consenso mexicano para el diagnóstico y tratamiento de la colitis ulcerosa crónica idiopática. Rev Gastroenterol Mex. 2018;83(2):144-167.

17. Ungaro R, Mehandru S, Allen PB, Peyrin-Biroulet L, Colombel JF. Ulcerative colitis. Lancet. 2017;389(10080):1756-1770.

18. Eisenstein M. Ulcerative colitis: towards remission. Nature. 2018;563(7730):S33. doi:10.1038/d41586-018-07276-2.

19. Adler E, Tabaa A, Kassam Z, Zydek M, Terdiman J, El-Nachef N. Capsule-Delivered Fecal Microbiota Transplant Is Safe and Well Tolerated in Patients with Ulcerative Colitis. Dig Dis Sci. 2019;64(9):2452-2454. doi:10.1007/s10620-019-05596-5.

20. Narula N, Kassam Z, Yuan Y, Colombel JF, Ponsioen C, Reinisch W, Moayyedi P. Systematic Review and Meta-analysis: Fecal Microbiota Transplantation for 
Treatment of Active Ulcerative Colitis. Inflamm Bowel Dis. 2017;23(10):1702-1709. doi:10.1097/MIB.0000000000001228.

21. Paramsothy S, Nielsen S, Kamm MA, Deshpande NP, Faith JJ, Clemente JC, Paramsothy R, Walsh AJ, van den Bogaerde J, Samuel D, Leong RWL, Connor S, Ng W, Lin E, Borody TJ, Wilkins MR, Colombel JF, Mitchell HM, Kaakoush NO. Specific Bacteria and Metabolites Associated With Response to Fecal Microbiota Transplantation in Patients With Ulcerative Colitis. Gastroenterology. 2019;156(5):1440-1454.e2. doi:10.1053/j.gastro.2018.12.001.

22. Paramsothy S, Kamm MA, Kaakoush NO, Walsh AJ, van den Bogaerde J, Samuel D, Leong RWL, Connor S, Ng W, Paramsothy R, Xuan W, Lin E, Mitchell HM, Borody TJ. Multidonor intensive faecal microbiota transplantation for active ulcerative colitis: a randomised placebo-controlled trial. Lancet. 2017;389(10075):1218-1228. doi:10.1016/S0140-6736(17)30182-4.

23. Mahajan R, Midha V, Singh A, Mehta V, Gupta Y, Kaur K, Sudhakar R, Singh Pannu A, Singh D, Sood A. Incidental benefits after fecal microbiota transplant for ulcerative colitis. Intest Res. 2020;18(3):337-340. doi: 10.5217/ir.2019.00108.

24. Tian Y, Zhou Y, Huang S, Li J, Zhao K, Li X, Wen X, Li XA. Fecal microbiota transplantation for ulcerative colitis: a prospective clinical study. BMC Gastroenterol. 2019;19(1):116. doi:10.1186/s12876-019-1010-4.

25. Uygun A, Ozturk K, Demirci H, Oger C, Avci IY, Turker T, Gulsen M. Fecal microbiota transplantation is a rescue treatment modality for refractory ulcerative colitis. Medicine (Baltimore). 2017;96(16):e6479. doi:10.1097/MD.0000000000006479.

26. Ballester Ferré MP, Boscá-Watts MM, Mínguez Pérez M. Crohn's disease. Enfermedad de Crohn. Med Clin (Barc). 2018;151(1):26-33. doi:10.1016/j.medcli.2017.10.036

27. Veauthier B, Hornecker JR. Crohn's Disease: Diagnosis and Management. Am Fam Physician. 2018;98(11):661-669.

28. Ibraheim H, Giacomini C, Kassam Z, Dazzi F, Powell N. Advances in mesenchymal stromal cell therapy in the management of Crohn's disease. Expert Rev Gastroenterol Hepatol. 2018;12(2):141-153. doi:10.1080/17474124.2018.1393332.

29. Gajendran M, Loganathan P, Catinella AP, Hashash JG. A comprehensive review and update on Crohn's disease. Dis Mon. 2018;64(2):20-57 doi:10.1016/j.disamonth.2017.07.001.

30. Wang H, Cui B, Li Q, Ding X, Li P, Zhang T, Yang X, Ji G, Zhang F. The Safety of Fecal Microbiota Transplantation for Crohn's Disease: Findings from A Long-Term Study. Adv Ther. 2018;35(11):1935-1944. doi:10.1007/s12325-018-0800-3.

31. Vaughn BP, Vatanen T, Allegretti JR, Bai A, Xavier RJ, Korzenik J, Gevers D, Ting A, Robson SC, Moss AC. Increased Intestinal Microbial Diversity Following Fecal Microbiota Transplant for Active Crohn's Disease. Inflamm Bowel Dis. 2016;22(9):2182-2190. doi:10.1097/MIB.0000000000000893.

32. Gutin L, Piceno Y, Fadrosh D, Lynch K, Zydek M, Kassam Z, LaMere B, Terdiman J, Ma A, Somsouk M, Lynch S, El-Nachef N. Fecal microbiota transplant for Crohn 
disease: A study evaluating safety, efficacy, and microbiome profile. United European Gastroenterol J. 2019;7(6):807-814. doi:10.1177/2050640619845986.

33. Li P, Zhang T, Xiao Y, Tian L, Cui B, Ji G, Liu YY, Zhang F. Timing for the second fecal microbiota transplantation to maintain the long-term benefit from the first treatment for Crohn's disease. Appl Microbiol Biotechnol. 2019;103(1):349-360. doi:10.1007/s00253-018-9447-x.

34. Sokol H, Landman C, Seksik P, Berard L, Montil M, Nion-Larmurier I, Bourrier A, Le Gall G, Lalande V, De Rougemont A, Kirchgesner J, Daguenel A, Cachanado M, Rousseau A, Drouet É, Rosenzwajg M, Hagege H, Dray X, Klatzman D, Marteau P; Saint-Antoine IBD Network, Beaugerie L, Simon T. Fecal microbiota transplantation to maintain remission in Crohn's disease: a pilot randomized controlled study. Microbiome. 2020;8(1):12. doi:10.1186/s40168-020-0792-5.

35. Herndon CC, Wang YP, Lu CL. Targeting the gut microbiota for the treatment of irritable bowel syndrome. Kaohsiung J Med Sci. 2020;36(3):160-170. doi:10.1002/kjm2.12154.

36. Enck P, Aziz Q, Barbara G, Farmer AD, Fukudo S, Mayer EA, Niesler B, Quigley EM, Rajilić-Stojanović M, Schemann M, Schwille-Kiuntke J, Simren M, Zipfel S, Spiller RC. Irritable bowel syndrome. Nat Rev Dis Primers. 2016;2:16014. doi:10.1038/nrdp.2016.14.

37. Camilleri M. Management Options for Irritable Bowel Syndrome. Mayo Clin Proc. 2018;93(12):1858-1872. doi:10.1016/j.mayocp.2018.04.032.

38. Mazzawi T, Lied GA, Sangnes DA, El-Salhy M, Hov JR, Gilja OH, Hatlebakk JG, Hausken T. The kinetics of gut microbial community composition in patients with irritable bowel syndrome following fecal microbiota transplantation. PLoS One. 2018;13(11):e0194904. doi:10.1371/journal.pone.0194904.

39. Mazzawi T, Hausken T, Hov JR, Valeur J, Sangnes DA, El-Salhy M, Gilja OH, Hatlebakk JG, Lied GA. Clinical response to fecal microbiota transplantation in patients with diarrhea-predominant irritable bowel syndrome is associated with normalization of fecal microbiota composition and short-chain fatty acid levels. Scand J Gastroenterol. 2019;54(6):690-699. doi:10.1080/00365521.2019.1624815.

40. Halkjær SI, Christensen AH, Lo BZS, Browne PD, Günther S, Hansen LH, Petersen AM. Faecal microbiota transplantation alters gut microbiota in patients with irritable bowel syndrome: results from a randomised, double-blind placebo-controlled study. Gut. 2018;67(12):2107-2115. doi:10.1136/gutjnl-2018-316434.

41. El-Salhy M, Hatlebakk JG, Gilja OH, Bråthen Kristoffersen A, Hausken T. Efficacy of faecal microbiota transplantation for patients with irritable bowel syndrome in a randomised, double-blind, placebo-controlled study. Gut. 2020;69(5):859-867. doi:10.1136/gutjnl-2019-319630.

42. Dai M, Liu Y, Chen W, Buch H, Shan Y, Chang L, Bai Y, Shen C, Zhang X, Huo Y, Huang D, Yang Z, Hu Z, He X, Pan J, Hu L, Pan X, Wu X, Deng B, Li Z, Cui B, Zhang F. Rescue fecal microbiota transplantation for antibiotic-associated diarrhea in critically ill patients. Crit Care. 2019;23:324. doi:10.1186/s13054-019-2604-5.

43. Singh R, de Groot PF, Geerlings SE, Hodiamont CJ, Belzer C, Berge IJMT, de Vos WM, Bemelman FJ, Nieuwdorp M. Fecal microbiota transplantation against intestinal 
colonization by extended spectrum beta-lactamase producing Enterobacteriaceae: a proof of principle study. BMC Res Notes. 2018 Mar 22;11(1):190. doi: 10.1186/s13104-018-3293-x.

44. Kakihana K, Fujioka Y, Suda W, Najima Y, Kuwata G, Sasajima S, Mimura I, Morita H, Sugiyama D, Nishikawa H, Hattori M, Hino Y, Ikegawa S, Yamamoto, Toya T, Doki N, Koizumi K, Honda K, Ohashi K. Fecal microbiota transplantation for patients with steroid-resistant acute graft-versus-host disease of the gut. Blood. 2016;128(16):2083-2088. doi: 10.1182/blood-2016-05-717652.

45. Ding X, Li Q, Li P, Chen X, Xiang L, Bi L, Zhu J, Huang X, Cui B, Zhang F. Fecal microbiota transplantation: A promising treatment for radiation enteritis? Radiother Oncol. 2020;143:12-18. doi:10.1016/j.radonc.2020.01.011. 